\title{
RISCO DE HIPOTERMIA PERIOPERATÓRIA: REVISÃO INTEGRATIVA
}

\author{
Risk of perioperative hypothermia: An integrative review
}

Riesgo de hipotermia perioperatoria: Una revisión integradora

Monique Alves Mendes ${ }^{1}$ (D), Natália Kaizer Rezende Ortega de Barros ${ }^{1}$ (D), Thalita Gomes do Carmo ${ }^{2 *}$ (i)

RESUMO: Objetivo: Identificar a ocorrência e os fatores de risco determinantes para o desenvolvimento do diagnóstico de enfermagem risco de hipotermia perioperatória. Método: Revisão integrativa, usando o acrônimo PIO (pacientes, intervenção, outcomes/desfecho), em que P=pacientes adultos e idosos; $\mathrm{I}=$ cirurgias eletivas; $\mathrm{O}=$ fatores de risco associados à hipotermia perioperatória. A revisão foi conduzida e reportada com base no Check-list Preferred Reporting Items for Systematic Reviews and Meta-Analyses (PRISMA), seguindo-se as sete etapas propostas pelo método PRISMA. Resultados: A busca gerou 854 artigos, sendo retirados os duplicados e os sem relevância. Após aplicação dos critérios de exclusão, 13 artigos foram submetidos à análise final. Índice de massa corporal baixo, idade avançada e tempo cirúrgico prolongado foram os fatores mais relacionados ao desenvolvimento de hipotermia nos pacientes cirúrgicos. O manejo da hipotermia colabora para diminuição dos riscos de eventos adversos cardíacos, complicações infecciosas, sangramentos e traz maior conforto para o paciente. Conclusão: O enfermeiro precisa agir antes do quadro hipotérmico se instalar, reconhecendo os fatores de risco inerentes ao paciente e identificando quais tecnologias do cuidado aplicar.

Palavras-chave: Enfermagem perioperatória. Diagnóstico de Enfermagem. Hipotermia. Processo de Enfermagem. Cuidados intraoperatórios.

ABSTRACT: Objective: To identify the occurrence and the risk factors that determine the development of the nursing diagnosis 'risk for perioperative hypothermia'. Method: Integrative review, using the acronym PIO (patients, intervention, outcomes), in which P=adult and older adults; $\mathrm{I}=$ elective surgeries; $\mathrm{O}=$ risk factors associated with perioperative hypothermia. The review was conducted and reported based on the Check-list Preferred Reporting Items for Systematic Reviews and Meta-Analyzes (PRISMA), following the seven steps proposed by the PRISMA method. Results: The search generated 854 articles, excluding duplicates and non-relevant titles. After applying the exclusion criteria, 13 articles were submitted to the final analysis. Low body mass index, advanced age and prolonged surgical time were the factors most related to the development of hypothermia in surgical patients. The management of hypothermia helps to reduce the risk of adverse cardiac events, infectious complications, and bleeding, besides bringing greater comfort to patients. Conclusion: Nurses need to act before the hypothermic condition sets in, recognizing the risk factors inherent to each patient and identifying which care technologies to apply. Keywords: Perioperative nursing. Nursing diagnosis. Hypothermia. Nursing process. Intraoperative care.

RESUMEN: Objetivo: Identificar la ocurrencia y los factores de riesgo que determinan el desarrollo del Diagnóstico de Enfermería Riesgo de Hipotermia Perioperatoria. Método: Revisión integrativa, utilizando el acrónimo PIO (Pacientes, Intervención, Outcomes / Resultado), donde P=pacientes adultos y ancianos; I=cirugías electivas; $\mathrm{O}=$ factores de riesgo asociados a hipotermia perioperatoria. La revisión se llevó a cabo y se informó utilizando los elementos de informe preferidos de la lista de verificación para revisiones sistemáticas y metaanálisis (Check-list Preferred Reporting Items for Systematic Reviews and Meta-Analyses - PRISMA), siguiendo los siete pasos propuestos por el método PRISMA. Resultados: La búsqueda generó 854 artículos, eliminando los duplicados y los no relevantes. Tras aplicar los criterios de exclusión, se sometieron 13 artículos al análisis final. El bajo índice de masa corporal, la edad avanzada y el tiempo quirúrgico prolongado fueron los factores más relacionados con el desarrollo de hipotermia en los pacientes quirúrgicos. El manejo de la hipotermia ayuda a reducir los riesgos de eventos cardíacos adversos, complicaciones infecciosas, hemorragias y brinda mayor comodidad al paciente. Conclusión: La enfermera debe actuar antes de que se presente la condición hipotérmica, reconociendo los factores de riesgo inherentes al paciente e identificando qué tecnologías de atención aplicar. Palabras clave: Enfermería perioperatoria. Diagnóstico de enfermería. Hipotermia. Proceso de enfermería. Cuidados intraoperatorios.

'Acadêmica de Enfermagem da Universidade Federal Fluminense - Niterói (RJ), Brasil.

Enfermeira. Professora adjunta da Universidade Federal Fluminense - Niterói (RJ), Brasil.

*Autora correspondente: thalitacarmo@id.uff.b

Recebido: 19/08/2020 - Aprovado: 21/11/2020

https://doi.org/10.5327/Z1414-4425202100010009 


\section{INTRODUÇÃo}

Estima-se que a cada ano 63 milhões de pessoas sejam submetidas a tratamentos cirúrgicos por injúrias traumáticas, que outras 10 milhões de operações sejam realizadas por complicações relacionadas à gravidez e mais 31 milhões para tratar malignidades ${ }^{1}$. Muito embora os procedimentos tenham a intenção de salvar vidas, falhas no processo de assistência cirúrgica podem causar danos consideráveis, ressaltando que cerca de metade deles poderia ser evitado, cabendo à equipe de cuidados perioperatórios melhorar a comunicação e reduzir os danos ao paciente.

Além disso, a assistência cirúrgica vem sendo componente essencial da assistência em saúde pelo mundo por quase um século. À medida que as incidências de injúrias traumáticas, cânceres e doenças cardiovasculares continuem a aumentar, o impacto da intervenção cirúrgica nos sistemas de saúde pública também crescerá. Contudo a falta de acesso à assistência cirúrgica de alta qualidade vem sendo um problema significativo, ainda que as intervenções cirúrgicas possam ser proveitosas no que diz respeito às vidas salvas e às incapacidades evitadas ${ }^{1}$.

A prevenção e o tratamento da hipotermia são de extrema importância para impedir aumento da morbidade relacionada a eventos cardíacos adversos, infecção do sítio cirúrgico, prejuízos na função plaquetária, alteração no metabolismo de proteínas, alteração no metabolismo de fármacos, variações nos níveis séricos de potássio, vasoconstrição periférica, redução da tensão de oxigênio subcutâneo, anormalidades da coagulação, entre outros prejuízos ${ }^{2}$.

Segundo a North American Nursing Diagnosis Association (NANDA-I), o diagnóstico de enfermagem risco de hipotermia perioperatória foi aprovado em 2013, sendo revisado em 2017, publicado em diversos países e em vários idiomas ${ }^{3}$, demonstrando a importância de aprofundar a comprovação da validade do diagnóstico na prática clínica de enfermagem, comparando diferentes locais, participantes ou intervenções, também especificando meios de medida da efetividade, eficácia e dos custos relacionados a possíveis complicações de saúde por causa da hipotermia perioperatória.

O diagnóstico de enfermagem (00254) risco de hipotermia perioperatória (2013, 2017, nível de evidência 2.1) tem como definição "suscetibilidade a uma queda inadvertida na temperatura corporal central abaixo de $36^{\circ} \mathrm{C}$, que ocorre no período entre 1 hora antes até 24 horas após cirurgia, que pode comprometer a saúde"3. Encontra-se inserido na estrutura taxonômica no domínio 11, segurança e proteção, classe 6, termorregulação ${ }^{3}$.
Desse modo, pode-se destacar como fatores de risco, ou seja, determinantes para o desenvolvimento da hipotermia perioperatória: "Baixa temperatura ambiental; Transferência excessiva de calor por condução; Transferência excessiva de calor por convexão; Transferência excessiva de calor por radiação"3. Logo, é um diagnóstico que permite pensar em classificar pacientes com ou sem risco, antever complicações, possibilitar intervenção precoce, estimar prevalências e incidências ${ }^{3}$.

A população com risco de desenvolvimento da hipotermia perioperatória trata-se do conjunto de indivíduos que possuem uma ou mais características que aumentam a probabilidade de desenvolver a hipotermia perioperatória, tais como: "Baixa temperatura no pré-operatório $\left(<36^{\circ} \mathrm{C}\right)$; Baixo peso corporal; Escore de classificação do estado físico da American Society of Anesthesiologists (ASA) $>1$ "’3. É um diagnóstico que leva a refletir sobre medidas preventivas antes, durante e depois do ato cirúrgico, situação na qual o paciente estará mais propenso a apresentar risco de hipotermia.

De acordo com um estudo da Association of periOperative Registered Nurses (AORN), os enfermeiros devem estar alertas aos fatores demográficos (exemplo: idade, sexo) e aos fatores cirúrgicos (exemplo: tipo de cirurgia) 4 . Além disso, anemias, sepses e mortalidades pós-operatórias são fatores significantemente associados à hipotermia. Com isso, os enfermeiros perioperatórios devem entender os fatores de risco e as complicações associadas à hipotermia perioperatória, com o intuito de desenvolver e testar iniciativas baseadas em evidências, aperfeiçoando o atendimento e promovendo melhores resultados aos pacientes ${ }^{4}$.

Como condições associadas, apresentam-se: "Anestesia local e geral combinadas; Complicações cardiovasculares; Neuropatia diabética; Procedimento cirúrgico"”. O aprofundamento dessas questões tem a finalidade de melhor entender o diagnóstico e sua influência na recuperação dos pacientes no pós-operatório. E, principalmente, contribuir para efetiva implementação de cuidados de qualidade, como influenciador na diminuição dos custos em saúde e na recuperação plena dos pacientes, com o intuito de garantir sua segurança.

Por meio de um estudo de validação, comprova-se a existência de um indicador/fator determinante do diagnóstico e analisa-se, de certa forma, a construção de características que melhor definem as manifestações clínicas existentes, os fatores contribuintes causais daquele diagnóstico e os fatores de risco predisponentes do fenômeno, incentivando, assim, a prática de enfermagem baseada em evidências. 
O presente estudo pode auxiliar na melhora da assistência prestada pelos enfermeiros perioperatórios e também se faz relevante para as instituições prestadoras desse tipo de cuidado, pois a hipotermia aumenta o risco de complicações intra e pós-operatórias, aumentando o tempo de internação e os gastos da instituição.

\section{OBJETIVO}

Identificar a ocorrência e os fatores de risco determinantes para o desenvolvimento do diagnóstico de enfermagem risco de hipotermia perioperatória.

\section{MÉTODO}

Trata-se de uma revisão integrativa conduzida, de acordo com a ferramenta Preferred Reporting Items for Systematic Reviews and Meta-Analyses (PRISMA — Statement) ${ }^{5}$, baseada em sete principais etapas:

- construção de um protocolo de pesquisa;

- formulação da pergunta na prática baseada em evidência (PBE), utilizando o acrônimo PIO (pacientes, intervenção, outcomes/desfecho);

- definição dos descritores, estratégias de busca em cada uma das bases de dados selecionadas pelo pesquisador devendo ser variadas;

- determinação, seleção e revisão dos critérios de inclusão e exclusão;

- avaliação crítica dos estudos;

- coleta de dados utilizando instrumentos que analisem em pares (dois pesquisadores simultaneamente);

- síntese dos resultados/dados agrupados por semelhança.

\section{Critérios de elegibilidade}

Para atender à primeira etapa da revisão, os pesquisadores elaboraram um protocolo de pesquisa, no qual foram incluídos: a forma como os estudos seriam encontrados, os critérios de inclusão e exclusão dos artigos, a definição dos desfechos de interesse, a verificação da acurácia dos resultados e a determinação da qualidade dos estudos.

Na segunda etapa, realizou-se a construção da pergunta de pesquisa, recorrendo-se à utilização da estratégia PICO, que representa um acrônimo para pacientes, intervenção, comparação e outcomes (desfecho). Nesta pesquisa, como não houve comparação, utilizou-se o PIO, em que o $\mathrm{P}=$ paciente / problema, $\mathrm{I}=$ intervenção, $\mathrm{O}=$ outcome $/$ resultado/desfecho:

$\mathrm{P}$ - pacientes adultos e idosos;

I - cirurgias eletivas;

$\mathrm{O}$ - fatores de risco associados à hipotermia perioperátoria.

Formulou-se, então, a seguinte pergunta de pesquisa: quais são os fatores de risco associados à hipotermia perioperatória em pacientes adultos submetidos a cirurgias eletivas?

\section{Fontes de informação}

A terceira etapa foi o estabelecimento dos descritores traçados com base nos tesauros Medical Subject Headings MESH (PubMed), Descritores em Ciências da Saúde - DeCS (Literatura Latino-Americana e do Caribe em Ciências da Saúde - LILACS), TreeTerm (EMBASE) e Cumulative Index to Nursing and Allied Health Literature (CINAHAL): "adulto", "idoso", "fatores de risco", "procedimentos cirúrgicos operatórios", "hipotermia”, "período perioperatório", "adultos", "idosos", "procedimentos cirúrgicos eletivos"; tanto em português quanto em inglês ("adult", "middle-aged", "aged", "aged, 80 and over", "risk factors", "surgical procedures, operative", "hypothermia", "perioperative period") e associados entre si. Como estratégia de busca, utilizaram-se os operadores booleanos OR e AND para as associações.

As buscas on-line nas bases de dados Medical Literature and Retrieval System Online (MEDLINE) via PubMed, LILACS via BVS, CINAHAL e EMBASE via Elsevier iniciaram-se respectivamente nos dias 18 de fevereiro de 2020, 06 de março de 2020 e 11 de março de 2020.

$\mathrm{Na}$ quarta etapa, ocorreu a determinação, a seleção e a revisão dos critérios de inclusão e exclusão. Os critérios de inclusão do estudo foram: artigos que abordem hipotermia perioperatória em adultos e idosos. E os critérios de exclusão foram: artigos sem metodologia clara, artigos que não abordem fatores de risco, estudos de fontes secundárias, guidelines, protocolos de pesquisa, teses, dissertações e notas prévias. Como filtros, consideraram-se estudos publicados entre os anos 2015 e 2020, idade (adultos e idosos), tipo de publicação (artigos) e os idiomas português e inglês.

Na quinta etapa, realizou-se a avaliação crítica dos estudos. Os trabalhos científicos foram selecionados de acordo com seus conceitos, coletados por meio de um instrumento, com os seguintes itens: dados de identificação do artigo (autores e ano de publicação), país, base de dados onde foi encontrado, qualidade dos estudos quanto ao nível de evidência, 
metodologia e fatores de risco para hipotermia perioperatória (Figura 1).

Os estudos incluídos foram classificados de acordo com o nível de evidência e a força de recomendação, propostos pelo Centro de Medicina Baseada em Evidências (CEBM), Oxford, 2009 (Quadros 1 e 2).

Na sexta etapa, realizou-se a coleta de dados utilizando instrumentos, em planilha Excel, para análise por pares (dois pesquisadores simultaneamente) dos estudos conforme os critérios de inclusão e exclusão adotados.

Na sétima e última etapa, houve a interpretação e a discussão dos resultados, destacando-se os trabalhos que trouxeram, de forma mais clara e concisa, os fatores de risco para hipotermia perioperatória. Em seguida, apresentaram-se a revisão e a síntese do conhecimento produzido acerca dos desfechos estabelecidos.

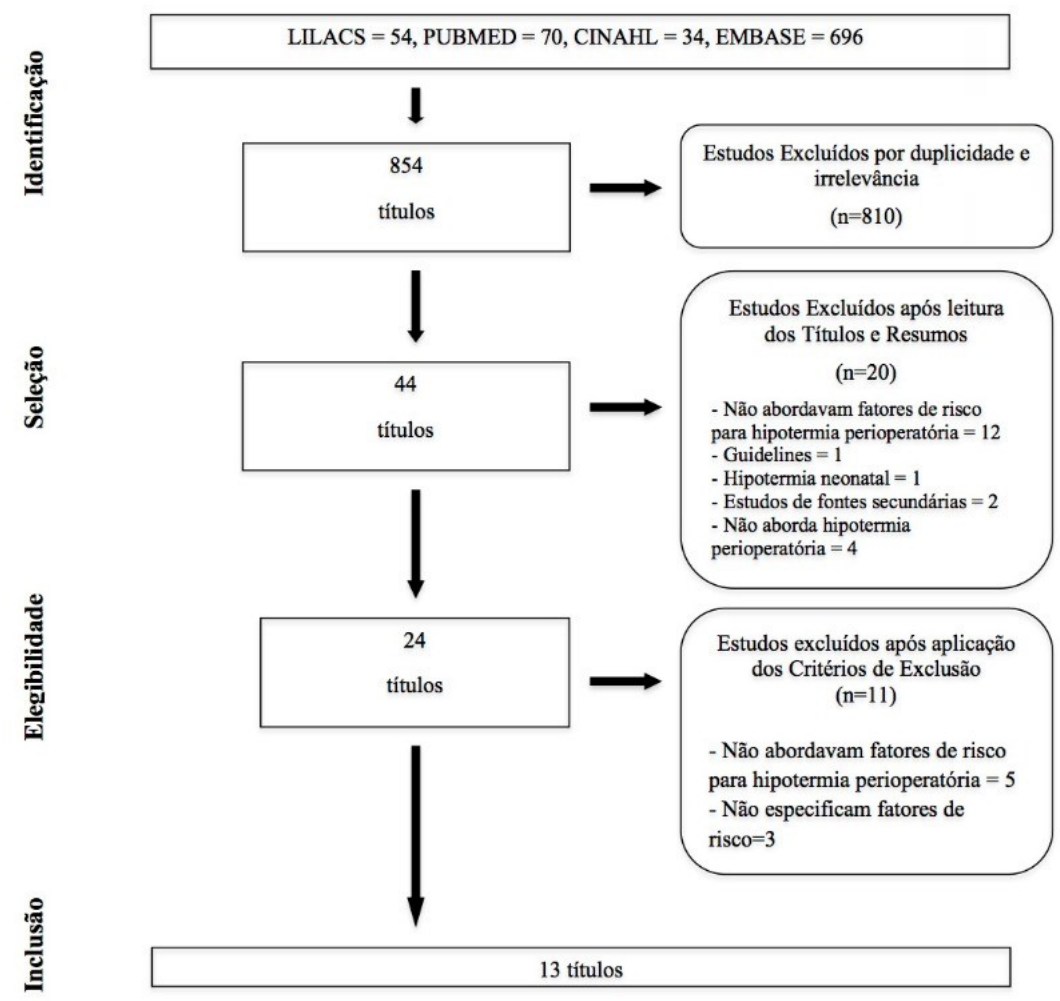

Figura 1. Fluxograma do processo de seleção e inclusão dos estudos segundo o Preferred Reporting Items for Systematic Reviews and Meta-Analyses (PRISMA 2009). Niterói, Rio de Janeiro, 2020.

Quadro 1. Nível de evidência para estudos de tratamento - Oxford Centre for Evidence-based Medicine.

Classificação

$1 \mathrm{~A}$

$1 \mathrm{~B}$

$2 \mathrm{~A}$

2B

$2 \mathrm{C}$

$3 \mathrm{~A}$

$3 \mathrm{~B}$

4

5

\section{Tipo de estudo}

Revisão sistemática (RS) de ensaios clínicos controlados e randomizados (ECR) homogêneos e de boa qualidade metodológica.

ECR com pequeno intervalo de confiança.

RS com homogeneidade de estudos de coorte.

Estudos de coorte; ECR com baixa qualidade metodológica.

Estudos observacionais de estudos terapêuticos (outcome research) e estudos ecológicos.

RS com homogeneidade de estudos de caso controle.

Estudos de caso controle.

Série de casos; estudos de coorte ou caso controle de baixa qualidade metodológica.

Opinião de especialista sem avaliação crítica.

Fonte: adaptação do Centro de Medicina Baseada em Evidências (CEBM). Tradução livre dos autores. 


\section{RESULTADOS}

A Figura 1 detalha o fluxograma de seleção dos artigos, desde a busca inicial até a inclusão final de estudos. A estratégia de busca utilizada gerou 854 publicações, sendo 54 na LILACS, 70 na PubMed, 34 na CINAHL, 696 na EMBASE. Excluíram-se 810 artigos por duplicidade e irrelevância. Quando analisado o título e o resumo, excluíram-se 20. Após leitura dos artigos na íntegra e aplicação dos critérios de exclusão, verificou-se que 11 não apresentavam relação com a temática, sendo excluídos. Assim, consideraram-se para análise final 13 artigos, que, portanto, compuseram a mostra deste estudo.

No Quadro 3, são apresentados os 13 artigos incluídos no estudo, destacando-se autores, ano, país onde o estudo foi desenvolvido, base de dados, nível de evidência, método e fatores de risco para hipotermia perioperatória. Dos 13 artigos incluídos no estudo, 8 estavam disponíveis na base de dados EMBASE e 5 na PubMed. Os anos de publicação variam de 2016 a 2020, sendo um artigo publicado em 2020, cinco em 2019, três em 2018, dois em 2017 e dois em 2016.
Os estudos mais antigos foram "Warming before and after epidural block before general anaesthesia for major abdominal surgery prevents perioperative hypothermia" e "Effect of perioperative inadvertent hypothermia on the ECG parameters in patients undergoing transurethral resection", publicados em 2016, e o mais recente, "Prevalence and multivariable factors associated with inadvertent intraoperative hypothermia in video-assisted thoracoscopic surgery: a single-center retrospective study", publicado em 2020.

Encontraram-se publicações em nove países diferentes. A Alemanha foi o país com maior número de artigos relacionados ao tema, totalizando três publicações. Estados Unidos e Canadá tiveram duas publicações cada. China, Brasil, França, Espanha, Grécia e Turquia tiveram um estudo incluído. Em relação ao idioma, todos os estudos são na língua inglesa. A maioria dos estudos é do tipo observacional.

Todos os estudos analisados realizaram exposição dos fatores intrínsecos e extrínsecos aos pacientes e suas correlações com a incidência e a prevalência de hipotermia no período perioperatório.

Quadro 2. Grau de Recomendação - Oxford Centre for Evidence-based Medicine.

Classificação

A

B

C

D
Tipo de estudo

Estudos nível 1 com resultados consistentes.

Exemplo: revisão Sistemática (RS) de ensaios clínicos controlados e randomizados (ECR).

Estudos nível 2 ou 3 com resultados consistentes.

Exemplo: RS de coorte; coorte; outcome research; estudos ecológicos; RS de caso controle e estudos de caso controle.

Série ou relatos de caso.

Opinião de especialista ou estudos classificados em qualquer outro nível que apresente resultados inconsistentes ou inconclusivos.

Fonte: adaptação do Centro de Medicina Baseada em Evidências (CEBM). Tradução livre dos autores.

Quadro 3. Artigos selecionados como amostra, segundo autores, ano, país de origem, base de dados, nível de evidência, método e fatores de risco para hipotermia.

\begin{tabular}{|c|c|c|c|c|c|c|}
\hline Autor & Ano & País & $\begin{array}{l}\text { Base de } \\
\text { dados }\end{array}$ & $\begin{array}{l}\text { Nível de } \\
\text { Evidência }\end{array}$ & Método & $\begin{array}{l}\text { Fatores de risco para } \\
\text { hipotermia perioperatória }\end{array}$ \\
\hline $\begin{array}{l}\text { Akers } \\
\text { et al. }{ }^{4}\end{array}$ & 2019 & $\begin{array}{l}\text { Estados } \\
\text { Unidos da } \\
\text { América }\end{array}$ & EMBASE & 3B & $\begin{array}{c}\text { Observacional } \\
\text { Transversal Retrospectivo } \\
\text { Documental }\end{array}$ & $\begin{array}{l}\text { Idade }>60 \text { anos; Colectomia; } \\
\text { Histerectomia; Colecistectomia } \\
\text { laparoscópica; Reparo de hérnia. }\end{array}$ \\
\hline Li et al. ${ }^{6}$ & 2020 & China & EMBASE & $3 B$ & $\begin{array}{c}\text { Observacional } \\
\text { Transversal Retrospectivo } \\
\text { Documental }\end{array}$ & $\begin{array}{l}\text { Idade } 51 \text { e } 66 \text { anos; IMC }<24 \text { kg/m²; } \\
\text { Duração da cirurgia }>2 \mathrm{~h} ; \\
\text { Cirurgias no turno da manhã (8h às } 14 \mathrm{~h}) ; \\
\text { Anestesia geral combinada. }\end{array}$ \\
\hline $\begin{array}{l}\text { Kleimeyer } \\
\text { et al. }{ }^{7}\end{array}$ & 2018 & $\begin{array}{l}\text { Estados } \\
\text { Unidos da } \\
\text { América }\end{array}$ & EMBASE & $3 B$ & $\begin{array}{c}\text { Observacional } \\
\text { Transversal Retrospectivo } \\
\text { Documental }\end{array}$ & $\begin{array}{c}\text { Sexo feminino; Baixo IMC }(<18,5 \mathrm{~kg} / \\
\left.\mathrm{m}^{2}\right) \text {; Idade avançada (média }=51,4 \text { anos); } \\
\text { Artroscopia de quadril; Baixa temperatura } \\
\text { pré-operatória (mínima de } 36^{\circ} \mathrm{C} \text { ). }\end{array}$ \\
\hline
\end{tabular}


Quadro 3. Continuação.

\begin{tabular}{|c|c|c|c|c|c|c|}
\hline Autor & Ano & País & $\begin{array}{l}\text { Base de } \\
\text { dados }\end{array}$ & $\begin{array}{l}\text { Nível de } \\
\text { Evidência }\end{array}$ & Método & $\begin{array}{l}\text { Fatores de risco para } \\
\text { hipotermia perioperatória }\end{array}$ \\
\hline $\begin{array}{l}\text { Emmert } \\
\text { et al. }{ }^{8}\end{array}$ & 2018 & Alemanha & PUBMED & $3 B$ & $\begin{array}{c}\text { Observacional } \\
\text { Transversal Retrospectivo } \\
\text { Documental }\end{array}$ & $\begin{array}{l}\text { Tempo de indução da anestesia (média } \\
\text { = } 75,26 \text { min); Menor área de superfície } \\
\text { corporal (média = 1,92 m); Infusão de fluidos } \\
\text { no intraoperatório; Toracotomia; Cateter } \\
\text { peridural; IMC baixo }\left(<18,5 \mathrm{~kg} / \mathrm{m}^{2}\right) .\end{array}$ \\
\hline $\begin{array}{l}\text { Mendonça } \\
\text { et al. }{ }^{9}\end{array}$ & 2019 & Brasil & EMBASE & $2 B$ & $\begin{array}{l}\text { Observacional } \\
\text { Longitudinal Prospectivo } \\
\text { Multicêntrico }\end{array}$ & $\begin{array}{l}\text { Idosos (média = } 70 \text { anos); } \\
\text { Anestesia combinada. }\end{array}$ \\
\hline $\begin{array}{l}\text { Chalari } \\
\text { et al. }{ }^{10}\end{array}$ & 2019 & Grécia & EMBASE & $1 \mathrm{~A}$ & $\begin{array}{l}\text { Ensaio clínico } \\
\text { randomizado prospectivo }\end{array}$ & $\begin{array}{l}\text { Ressecção Transuretral em Solução Salina } \\
\text { (TURi); Ressecção transuretral (TUR); IMC } \\
<26,9 \mathrm{~kg} / \mathrm{m}^{2} \text {; Idade avançada >86 anos. }\end{array}$ \\
\hline $\begin{array}{l}\text { Bayir } \\
\text { et al."1 }\end{array}$ & 2016 & Turquia & PUBMED & $2 C$ & $\begin{array}{c}\text { Observacional } \\
\text { Longitudinal Prospectivo }\end{array}$ & $\begin{array}{l}\text { Ressecção transuretral de próstata; } \\
\text { Ressecção transuretral de bexiga. }\end{array}$ \\
\hline $\begin{array}{l}\text { Alfonsi } \\
\text { et al. } .^{12}\end{array}$ & 2019 & França & EMBASE & $2 B$ & $\begin{array}{l}\text { Observacional } \\
\text { Prospectivo Multicêntrico }\end{array}$ & $\begin{array}{l}\text { Idade } \geq 70 \text { anos; Duração da anestesia ( } 1 \text { a } \\
\text { 2h); Diminuição na temperatura de }>0,5^{\circ} \mathrm{C} \\
\text { indução da anestesia e incisão cirúrgica. }\end{array}$ \\
\hline $\begin{array}{l}\text { Becerra } \\
\text { et al. }{ }^{13}\end{array}$ & 2019 & Espanha & EMBASE & $2 \mathrm{C}$ & $\begin{array}{c}\text { Observacional } \\
\text { Longitudinal Prospectivo }\end{array}$ & $\begin{array}{l}\text { Ressecção transuretral (TUR); Sexo } \\
\text { masculino; Infusão de líquidos em } \\
\text { temperatura ambiente. }\end{array}$ \\
\hline $\begin{array}{l}\text { Horn } \\
\text { et al. }{ }^{14}\end{array}$ & 2016 & Alemanha & PUBMED & $1 \mathrm{~A}$ & $\begin{array}{l}\text { Ensaio clínico } \\
\text { randomizado prospectivo }\end{array}$ & $\begin{array}{l}\text { Longa duração de cirurgias abdominais } \\
\qquad(\geq 120 \mathrm{~min}) .\end{array}$ \\
\hline $\begin{array}{l}\text { Ziegler } \\
\text { et al. }{ }^{15}\end{array}$ & 2019 & Alemanha & EMBASE & $3 B$ & $\begin{array}{l}\text { Observacional } \\
\text { Transversal Retrospectivo } \\
\text { Documental }\end{array}$ & $\begin{array}{l}\text { Sedativos; Ventilação mecânica; } \\
\text { Queimadura extensa; Lesão por inalação; } \\
\text { Queimaduras } 3^{\circ} \text { grau. }\end{array}$ \\
\hline $\begin{array}{l}\text { Ziolkowski } \\
\text { et al. }{ }^{16}\end{array}$ & 2017 & Canadá & PUBMED & $3 B$ & $\begin{array}{l}\text { Observacional } \\
\text { Longitudinal } \\
\text { Restrospectivo } \\
\text { Documental }\end{array}$ & $\begin{array}{c}\text { Tempo de cirurgia } \\
\geq 3 \mathrm{~h}\end{array}$ \\
\hline $\begin{array}{l}\text { Desgranges } \\
\text { et al. }{ }^{17}\end{array}$ & 2017 & Canadá & PUBMED & $2 C$ & $\begin{array}{c}\text { Observacional } \\
\text { Longitudinal Prospectivo }\end{array}$ & $\begin{array}{l}\text { Volume total de fluidos infundidos } 650 \mathrm{~mL} \text {; } \\
\text { Temperatura do paciente na chegada à } \\
\text { sala de cirurgia }<37,1^{\circ} \mathrm{C} \text {; Temperatura do } \\
\text { paciente na incisão na pele }<36,6^{\circ} \mathrm{C} \text {. }\end{array}$ \\
\hline
\end{tabular}

\section{DISCUSSÃO}

A maioria dos artigos incluídos neste estudo citou o baixo índice de massa corporal (IMC), a idade avançada e o tempo cirúrgico prolongado como fatores importantes para o desenvolvimento da hipotermia ${ }^{4,6-10,12}$. Um estudo citou o uso de dispositivos de aquecimento por 15 minutos antes da cirurgia como procedimento importante na prevenção da hipotermia ${ }^{12}$. A cirurgia de ressecção transuretral de próstata foi a mais relacionada a situações de hipotermia pós-operatória ${ }^{10-13}$.
Um estudo retratou que a prevalência da hipotermia em cirurgias de toracoscopia videoassistida era de $72,7 \%$ e estes casos estavam correlacionados a fatores de risco como: idade avançada, tempo de preparação, cirurgias longas, baixa temperatura do ambiente, cirurgias que ocorriam pela manhã e combinação de anestesia geral com bloqueio paravertebral antes da intubação. O sobrepeso mostrou-se um fator de proteção contra a hipotermia ${ }^{6}$.

Além dos fatores de risco citados, outros autores identificaram a temperatura baixa no pré-operatório, o sexo feminino, 
o IMC baixo e os procedimentos de pelve e quadril ${ }^{7}$. Pessoas que possuem algum desses fatores de risco necessitam de intervenções específicas para prevenção da hipotermia e para limitar sua morbidez.

Observou-se que a hipotermia também é mais comum em pacientes que já tenham sido submetidos a cirurgias ortopédicas. A função pulmonar prejudicada foi relacionada com a hipotermia perioperatória ${ }^{8}$.

Em outra publicação, na qual os autores estudaram 78 pacientes, $69,2 \%$ apresentaram hipotermia no momento da admissão na recuperação pós-anestésica (RPA); desses, $65,4 \%$ estavam com a temperatura entre 34 e $35,9^{\circ} \mathrm{C}$ e $3,8 \%$ tinham temperatura menor que $34^{\circ} \mathrm{C}^{9}$. O grupo que apresentou hipotermia havia recebido predominantemente anestesia regional associada à morfina e ao sufentanil; já o grupo com normotermia havia recebido apenas fentanil. Os pacientes eram predominantemente do sexo masculino, com idades entre 30 e 70 anos e classificação ASA II e III. Nos idosos, a cirurgia predominante foi a ressecção transuretral de próstata, nos mais jovens, predominou a cirurgia cesariana. Quanto ao tipo de anestesia, os jovens foram submetidos à anestesia regional ou geral, enquanto a maioria dos idosos foi submetida à anestesia regional.

Pacientes submetidos à ressecção transuretral têm risco elevado para o desenvolvimento de hipotermia. Os casos de hipotermia nessas cirurgias foram ligeiramente maiores $(64,1 \%)$ do que nos que fizeram a ressecção transuretral de próstata $(60 \%)^{10}$. A temperatura no pós-operatório é consideravelmente menor do que a do pré-operatório em todos os pacientes. A redução da hipotermia colabora com a redução do risco de eventos adversos cardíacos ${ }^{11}$.

De acordo com um estudo multicêntrico conduzido na França, a queda da temperatura corporal ocorre após o processo de indução anestésica e permanece até a admissão na $\mathrm{RPA}^{12}$. Os autores enfatizaram que os sistemas de aquecimento, na maioria das vezes, são usados de forma pobre, em que o profissional simplesmente coloca a manta térmica no paciente, o que não é suficiente para prevenir a hipotermia perioperatória. É necessária a combinação de um pré-aquecimento antes da cirurgia somado ao aquecimento no intraoperatório. Portanto os profissionais devem fazer melhor uso desses mecanismos de aquecimento e também realizar a monitorização intermitente da temperatura do paciente para identificar precocemente a hipotermia.

O pré-aquecimento é uma técnica importante, realizada antes da operação. Foram relacionados os tempos de pré-aquecimento antes do procedimento de ressecção transuretral de próstata com anestesia espinhal. Os pacientes foram divididos em: grupo 1 (sem pré-aquecimento), grupo 2 (pré-aquecimento por 15 minutos), grupo 3 (pré-aquecimento por 30 minutos) e grupo 4 (pré-aquecimento por 45 minutos). Os pacientes dos grupos 1 e 4 apresentaram mais hipotermia ao fim do procedimento cirúrgico, $96,4 \%$ e $90,5 \%$, respectivamente. Os pacientes dos grupos 2 e 3 tiveram taxas semelhantes para desenvolver hipotermia (aproximadamente $74,0 \%)^{13}$.

Desse modo, o pré-aquecimento por 15 minutos mostrou-se mais efetivo do que tempos maiores de pré-aquecimento ou nenhum pré-aquecimento. Episódios de tremores na RPA foram observados em grande parte $(42,9 \%)$ nos pacientes que não sofreram pré-aquecimento e nos que sofreram aquecimento por 45 minutos (9,5\%); pacientes dos grupos 2 e 3 não apresentaram tremores. O tempo de permanência na RPA foi menor para os grupos 2 e 3, em comparação com os do grupo $1^{13}$. Em outro estudo, evidenciou-se o benefício do pré-aquecimento por 15 minutos antes da colocação do cateter epidural, sendo suficiente para evitar a hipotermia em todos os pacientes analisados ${ }^{14}$.

A hipotermia também é um fator desencadeante de piores desfechos em pacientes queimados, podendo refletir uma série de outros danos. A monitorização da temperatura deve ser feita com rigor especial nos pacientes queimados, pois neles a hipotermia determina a necessidade de maior atenção em seu tratamento primário ${ }^{15}$.

Pacientes com hipotermia tinham queimaduras maiores e maior incidência de problemas respiratórios. A hipotermia aumenta o risco de complicações infecciosas, como sepse, pneumonia, infecções de trato urinário e de lesões ${ }^{16}$.

Nas cirurgias cesarianas, a obesidade, o aquecimento ativo e a administração de ocitocina no momento do trabalho de parto são fatores associados à diminuição do risco de hipotermia. Já a infusão de mais de $650 \mathrm{~mL}$ de líquidos constituiu um fator de risco maior de ocorrência de hipotermia ${ }^{17}$.

Os enfermeiros têm papel primordial na prevenção da hipotermia, associando os fatores de risco do paciente e as complicações que a hipotermia perioperatória pode causar, focando suas intervenções em uma prática baseada em evidências, a fim de promover melhores desfechos aos pacientes cirúrgicos ${ }^{18}$.

Como limitação do estudo, podem-se citar os sistemas de busca, que são falhos e podem ter deixado de incluir estudos potencialmente elegíveis, bem como pelo fato de não terem sido feitas buscas em outras bases de dados. 


\section{CONCLUSÃO}

O presente estudo identificou a ocorrência e os fatores de risco determinantes para o desenvolvimento do diagnóstico de enfermagem risco de hipotermia perioperatória, expondo os fatores intrínsecos e extrínsecos que predispõem o paciente, suas correlações com a incidência e a prevalência de hipotermia no período perioperatório. Nos 13 artigos analisados, IMC baixo, idade avançada e tempo cirúrgico prolongado foram os fatores mais relacionados ao desenvolvimento de hipotermia nos pacientes cirúrgicos. O manejo da hipotermia colabora com a diminuição dos riscos de eventos adversos cardíacos, complicações infecciosas, sangramentos e traz maior conforto térmico para o paciente.

Faz-se necessário promover o conhecimento dos enfermeiros no que tange à identificação dos fatores que ameaçam os pacientes em grupos de risco de hipotermia. É indispensável que o enfermeiro saiba agir antes mesmo do quadro de hipotermia se instalar, reconhecendo os fatores de risco inerentes ao paciente e sabendo quais tecnologias do cuidado aplicar para a redução dessa complicação cirúrgica tão frequente.

\section{REFERÊNCIAS}

1. Organização Mundial da Saúde. Segundo desafio global para segurança do paciente: Cirurgias seguras salvam vidas (orientações para cirurgia segura da OMS). Rio de Janeiro: Organização Pan-Americana da Saúde; Ministério da Saúde; Agência Nacional de Vigilância Sanitária; 2009.

2. Associação Brasileira de Enfermeiros de Centro Cirúrgico, Recuperação Anestésica e Centro de Material e Esterilização (SOBECC). Diretrizes de práticas em enfermagem cirúrgica e processamento de produtos para a saúde. 7ª ed. São Paulo: SOBECC; Barueri: Manole; 2017.

3. North American Nursing Diagnosis Association (NANDA). Diagnósticos de enfermagem da NANDA Internacional: definições e classificação (2018-2020). $11^{\text {a }}$ ed. Porto Alegre: Artmed; 2018.

4. Akers L, Dupnick AC, Hillman EL, Bauer AG, Kinker LM, Wonder $\mathrm{AH}$. Inadvertent perioperative hypothermia risks and postoperative complications: a retrospective study. AORN J. 2019;109(6):741-7. https://doi.org/10.1002/aorn.12696

5. Liberati A, Altman DG, Tetzlaff J, Mulrow C, Gotzsche P, Loannidis JPA, et al. The PRISMA statement for reporting systematic reviews and meta-analyses of studies that evaluate health care interventions: explanation and elaboration. PLoS Med. 2009;6(7):e1000100. https:// doi.org/10.1371/journal.pmed.1000100

6. Li Y, Liang H, Feng Y. Prevalence and multivariable factors associated with inadvertent intraoperative hypothermia in video-assisted thoracoscopic surgery: a single-center retrospective study. BMC Anesthesiol. 2020;20:25. https://doi.org/10.1186/s12871-020-0953-x

7. Kleimeyer JP, Harris AHS, Sanford J, Maloney WJ, Kadry B, Bishop $\mathrm{JA}$. Incidence and risk factors for postoperative hypothermia after orthopaedic surgery. J Am Acad Orthop Surg. 2018;26(24):e497-e503. https://doi.org/10.5435/jaaos-d-16-00742

8. Emmert A, Gries G, Wand S, Buentzel J, Bräuer A, Quintel M, et al. Association between perioperative hypothermia and patient outcomes after thoracic surgery: a single center retrospective analysis. Medicine . 2018;97(17):e0528. https://doi.org/10.1097/md.0000000000010528

9. Mendonça FT, De Lucena MC, Quirino RS, Govêia CS, Guimarães GMN. Risk factors for postoperative hypothermia in the post-anesthetic care unit: a prospective prognostic pilot study. Rev Bras Anestesiol. 2019;69(2):122-30. https://doi.org/10.1016/j.bjane.2018.11.002
10. Chalari E, Intas G, Zyga S, Fildissis G, Tolia M, Toutziaris C, et al. Perioperative inadvertent hypothermia among urology patients who underwent transurethral resection with either TURis or transurethral resection of the prostate method. Urologia. 2019;86(2):69-73. https:// doi.org/10.1177/0391560318758937

11. Bayir H, Yildiz I, Erdem F, Tekelioglu UY, Bilgi M, Kocoglu H. Effect of perioperative inadvertent hypothermia on the ECG parameters in patients undergoing transurethral resection. Eur Rev Med Pharmacol Scie. 2016;20(8):1445-9.

12. Alfonsi $P$, Bekka $S$, Aegerter P. Prevalence of hypothermia on admission to recovery room remains high despite a large use of forcedair warming devices: findings of a non-randomized observational multicenter and pragmatic study on perioperative hypothermia prevalence in France. Plos One. 2019;14(12):e0226038. https://doi. org/10.1371/journal.pone.0226038

13. Becerra A, Valencia L, Ferrando C, Villar J, Rodríguez-Pérez A. Prospective observational study of the effectiveness of prewarming on perioperative hypothermia in surgical patients submitted to spinal anesthesia. Sci Rep. 2019;9(1):16477. https://doi.org/10.1038/ s41598-019-52960-6

14. Horn EP, Bein B, Broch O, Iden T, Böhm R, Latz SK, et al. Warming before and after epidural block before general anaesthesia for major abdominal surgery prevents perioperative hypothermia. Eur J Anaesthesiol. 2016;33(5):334-40. https://doi.org/10.1097/ eja. 0000000000000369

15. Ziegler B, Kenngott T, Fischer S, Hundeshagen G, Hartmann B, Horter $\mathrm{J}$, et al. Early hypothermia as risk factor in severely burned patients: a retrospective outcome study. Burns. 2019;45(8):1895-900. https:// doi.org/10.1016/j.burns.2019.07.018

16. Ziolkowski N, Rogers AD, Xiong W, Hong B, Patel S, Trull B, et al. The impact of operative time and hypothermia in acute burn surgery. Burns. 2017;43(8):1673-81. https://doi.org/10.1016/j.burns.2017.10.001

17.Desgranges FP, Bapteste L, Riffard C, Pop M, Cogniat B, Gagey AC, et al. Predictive factors of maternal hypothermia during cesarean delivery: a prospective cohort study. Obstetric Anesthesia Digest. 2017;64(9):919-27. https://doi.org/10.1007/ s12630-017-0912-2 